\title{
The role of metabolic adaptation to nutrient stress in pancreatic cancer
}

\author{
Abhishek Derle ${ }^{1, \#}$, Maria Chiara De Santis ${ }^{1, \#}$, Luca Gozzelino ${ }^{1, \#}$, Edoardo Ratto ${ }^{1, \#}$ and Miriam Martini ${ }^{1, *}$ \\ ${ }^{1}$ Department of Molecular Biotechnology and Health Science, Molecular Biotechnology Center, University of Torino, Torino, Italy. \\ \# Contributed equally to this manuscript. \\ * Corresponding Author: \\ Miriam Martini, Department of Molecular Biotechnology and Health Science, Molecular Biotechnology Center, via Nizza 52, 10126, \\ Torino, Italy; Phone: +39-11-6706425; E-mail: miriam.martini@unito.it
}

\begin{abstract}
Pancreatic cancer is the fourth most common cause of cancerrelated mortality, with a dismal prognosis that has changed little over the past few decades. Despite extensive efforts in understanding the oncogenetics of this pathology, pancreatic cancer remained largely elusive. One of the main characteristics of pancreatic cancer is the reduced level of oxygen and nutrient perfusion, caused by the new matrix formation, through the activation of stromal cells (desmoplasia). This stromal reaction leads to metabolic adaptations in surviving tumor cells in order to cope with these challenging conditions. The oncogenic signaling driven by KRAS mutation is necessary to fuel pancreatic tumors by activating key metabolic processes, including enhanced glycolysis and glutamine consumption. Here we review our current understanding of the pancreatic cancer metabolism as well as discuss recent work pointing to the importance of various metabolic strategies as well as autophagy and macropinocytosis as critical nutrient supply pathways. The elucidation of these metabolic networks may highlight new opportunities to further develop novel therapeutic strategies.
\end{abstract}

doi: $10.15698 /$ cst2018.12.166

Received originally: 03.08.2018

in revised form: 09.10.2018,

Accepted 10.10.2018,

Published 15.11.2018.

Keywords: pancreatic cancer, nutrient stress, metabolic rewiring, autophagy, macropinocytosis.
Abbreviations:
$\alpha$-KG $-\alpha$-ketoglutarate,
GOT1 - Glutamic-oxaloacetic
transaminase,
mTOR-mammalian target of
rapamycin,
PDAC - pancreatic ductal
adenocarcinoma,
$P P P$ - pentose phosphate pathway,
TCA - tricarboxylic acid.

\section{INTRODUCTION}

Pancreatic cancer is the fourth leading cause of cancer death in the developed world [1]. The most common subtype of pancreatic cancer is pancreatic ductal adenocarcinomas (PDACs) that arise from the exocrine pancreas. The lack of progress in early diagnosis and effective therapies are the main reasons why improvements in PDAC death rates have been so scarce. An early event during malignant transformation is the acquisition of activating mutations in the KRAS oncogene which occurs in $>90 \%$ of PDAC patients [2]. PDACs are highly "addicted" to this oncogene for multiple parameters influencing tumor initiation, progression and maintenance as demonstrated using genetically engineered mouse (GEM) models and human PDAC cell lines [3]. Recent studies have shown that oncogenic KRAS promotes metabolic rewiring to support increased energetic and biosynthetic demand by reprogramming glucose/glutamine metabolism and increasing autophagy and macropinocytosis $[4,5]$.
Autophagy is an evolutionarily conserved membranemediated process that delivers cytoplasmic constituents to lysosomes for degradation and component recycling. This process is triggered by nutrient shortage, protein damage, and oxidative stress occurring through the inhibition of the mammalian target of rapamycin (mTOR) pathway.

In addition to autophagy, cancer cells can also absorb extracellular components through an endocytic process of macropinocytosis. Macropinocytosis is a non-specific bulk internalization of large portions of extracellular fluid [6]. Firstly, the plasma membrane protrudes through actin filaments polymerization and entraps extracellular fluid. Subsequently, it folds inwards and by fusing with the basal membrane, it forms a large endocytic vesicle, the macropinosome. Finally, these macropinosomes undergo different steps of modification to shrink and concentrate their contents and are recycled to the cell membrane or fused with the lysosome for degradation. The latter mechanism is fundamental in cell metabolism since it permits the release 
of digested endocytosed material, predominantly in the form of aminoacyls and lipids [7].

This review gathers the recent findings on the role of metabolic adaptation to nutrient stress that cancer cells undergo during the development of pancreatic cancer. Importantly, we will focus on the currently available and potential therapeutic strategies aimed at improving the treatment of this life-threatening disease.

\section{PANCREATIC CANCER METABOLISM}

The metabolism of pancreatic cancer is largely adapted to support the high rate of proliferation and the synthesis of substrates necessary for tumor growth in its nutrientdeprived environment [5]. PDAC reprogramming is mainly driven by mutations in the oncogene KRAS [8-10]. In addition, other oncogenes and tumor suppressors, such as SMAD, Myc and p53, regulate the cellular metabolic state and are frequently mutated in PDAC $[2,9,11,12]$. The metabolic rewiring and different adaptation strategies implemented by PDAC are discussed below.

\section{Warburg effect}

The Warburg effect is one of the most established phenomenon observed in several tumors, in which cancer cells increase the rate of glycolysis even in the presence of oxygen [4], with glucose used not only as a mitochondrial source.

Several oncogenic mutations affect aerobic glycolysis. In pancreatic cancer, KRAS upregulates GLUT1 (glucose transporter 1), as well as HK1 (hexokinase 1), HK2 (hexokinase 2), PFK1 (phosphofructokinase 1) and LDHA (lactate dehydrogenase $A)$, which are key enzymes for the glycolytic processes (Figure 1). Nevertheless, the increased glycolytic flux is not accompanied by significant alterations in the amount of tricarboxylic acid (TCA) cycle intermediates, suggesting that glucose is primarily diverted to anabolic pathways, such as pentose phosphate pathway (PPP). The upregulation of LDHA pushes the conversion of pyruvate towards lactate, which can be secreted and taken up by more oxygenated neighbouring cells [13]. Hypoxic PDAC cells further promote the production of lactate by activating HIF-1, which negatively regulates the expression of pyruvate dehydrogenase [14]. The elevated rate of glycolysis promotes the shuttle of glycolytic intermediates towards the PPP. This pathway is composed by two distinct branches, called oxidative and non-oxidative, which contribute to fatty acid synthesis and NADPH production or nucleotide and glycolytic intermediate synthesis, respectively [15]. Moreover, p53 inhibits the glucose-6-phosphate dehydrogenase (G6PD), the first enzyme in the PPP pathway [11] and its loss in PDAC further promotes glucose consumption through the PPP pathway.

\section{Glutamine addiction}

Glutamine is considered as an essential amino acid for highly proliferating cells since it supports the synthesis of large amounts of metabolites. In the case of PDAC, it has been recently demonstrated that its metabolism is strongly dependent on glutamine as a replenishment for TCA cycle $[4,16,17]$. In the canonical pathway, glutamine enters the mitochondria and is converted to glutamate and subsequently to $\alpha$-ketoglutarate $(\alpha-K G)$ by the enzyme glutamate dehydrogenase 1 (GLUD1; Figure 1). $\alpha-K G$ is then metabolized in the TCA cycle and can stimulate cell growth through the mTORC1 pathway [18]. However, it has been demonstrated that oncogenic KRAS can downregulate GLUD1, favoring a non-canonical pathway for glutamine. The glutamine-derived glutamate is converted to aspartate by Glutamic oxaloacetic transaminase (GOT) 2, which is then released in the cytoplasm and sequentially metabolized by GOT1 (which is upregulated by KRAS) along with malate dehydrogenase (MDH1) and malic enzyme (ME1) to generate pyruvate and NADPH. This pathway is crucial to maintain the reducing power of PDAC cells, since it is the major source of NADPH. In fact, the oxidative branch of the PPP is strongly suppressed by KRAS, as previously described [9]. If glutamate metabolism is experimentally impaired, PDAC cells show reduced growth and increased oxidative stress [18]. Moreover, glutamine withdrawal or GOT2 inhibition may promote cell senescence through p27 pathway which is activated due to increased oxidative stress $[19,20]$. Interestingly, the fact that non-transformed cells are not affected by glutamine withdrawal, further demonstrates the addiction of PDAC cells to this pathway [21].

\section{Hypoxic adaptation}

In the last years, significant advances have been made to explain how PDAC enables metabolic reprogramming to maintain adequate intracellular nutrient levels despite limited external supply and promoting survival of cancer cells. To overcome low nutrient availability, PDAC cells overexpress several transporters to increase the efficiency of nutrient intake. The stabilization of HIF1, together with the oncogenic activity of KRAS and the loss of p53, leads to the upregulation of glucose transporters and glycolytic enzymes [4, 22]. Moreover, hypoxic cells produce and secrete more lactate, which contributes to the acidification of the tumor's microenvironment, dissolving adjacent fibers and facilitating tumor cells spread towards more oxygenated surroundings [23]. As described above, lactate can also be used by more oxygenated cells, giving rise to a symbiotic loop between hypoxic and normoxic cells [13]. Recent findings suggest that stromal cells can also support tumor growth by releasing non-essential amino acids [24]. In particular, stroma-associated pancreatic stellate cells are reported to secrete alanine, which can replace glucose as a source of carbon for the TCA cycle (Figure 1). Finally, some non-canonical strategies to increase nutrients availability are often upregulated in PDAC, such as macropinocytosis or autophagy, as described in the following paragraphs. The acquired dependence of PDAC on these pathways creates new vulnerabilities that can be therapeutically targeted. 


\section{AUTOPHAGY: THE CONUNDRUM OF PANCREATIC CANCER}

Autophagy is an intracellular homeostatic process which plays a crucial role in catabolic degradation of damaged cytoplasmic components by delivering them to lysosomes. Several studies indicate paradoxical role of this process in the pathogenesis of cancer, depending on tumor type and stage. On one hand, it exerts tumor suppressive functions during early stages of tumorigenesis $[25,26]$ by preventing the metabolic stress and chromosome instability. On the other hand, in already established tumor cells, it assists tumor growth and survival by providing an alternative source of energy and metabolic fuels $[27,28]$.

Recent reports exclusively points to a profound requirement of autophagy during advanced stages of PDAC progression in a cell autonomous manner. Loss of autophagy either by genetic means or by pharmacological inhibition suppresses pancreatic tumor cell growth in xenograft as well as GEM models [29]. A study conducted by Rosenfeldt, et al. has demonstrated that deletion of autophagy related genes (ATG), Atg5 and Atg7 in KRAS driven mouse models of pancreatic ductal adenocarcinoma, impedes progression of precursor lesions such as pre-malignant intraepithelial neoplasia (PanINs) to a late stage malignant tumor [30]. Similarly, tissue microarray data of PDAC patient samples reveals significant levels of autophagy related protein expression which, in turn, correlates to poor clinical outcomes [31]. One of the characteristic features of PDAC tumors is upregulation of autophagy under basal conditions and suppression of which averts further PDAC progression, thereby demonstrating the dependence of PDAC on this pathway [29].

PDAC exhibits extensive desmoplasia which is characterized by dense fibrotic stroma, poor vasculature and consequent harsh tumor micro-environmental conditions like hypoxia and nutrient deprivation [32]. Researchers hypothesize that autophagy acts as a salvage system to promote PDAC tumor survival and proliferation during these conditions by contributing to adaption strategies for different metabolic challenges. For example, in PANC-1 and MiaPaCa-2 cells, activation of autophagy through cell survival pathways like MAPK and NF-kB facilitates PDAC cell survival by inhibiting apoptosis [33]. Moreover, findings from two independent studies demonstrate that upregulation of autophagy in Panc-1 and BxPC-3 cells induced either by hypoxia-inducible factor-1 $\alpha$ (HIF- $1 \alpha$ ) or by long noncoding RNA metastasis-associated lung adenocarcinoma transcript 1 (MALAT1) promotes metastatic and proliferative ability of these cells [34, 35]. Autophagic genes and subsequent lysosomal systems are upregulated in PDAC. In physiological conditions of nutrient stress, autophagy onset is under control of MiT/TFE family of transcription factors (MITF, TFE3, TFEB and TFEC), however, during PDAC pathogenesis, nuclear retention of these proteins promotes activation of the autophagasome-lysosome machinery essential for PDA growth regardless of nutrient status [36, 37]. PDAC metabolism can be influenced by autophagy not only by providing metabolic substrates during the time of nutrient starva- tion but also by maintaining organelle functions (Figure 1). A recent study indicates that the key mitochondrial function of oxidative phosphorylation is maintained in an autophagy dependent mechanism [38]. Apart from this, autophagy has also been shown to promote PDAC tumor survival in non-cell-autonomous or cell-extrinsic manner i.e. by supporting the tumor metabolism by modulating other cell types or promoting cancer cachexia. [39]. Autophagy induced secretion of non-essential amino acids via pancreatic stellate cells helps in sustained survival of PDAC in an inhospitable environment [24]. Additionally, it also helps in maintaining pancreatic cancer stem cell activity [40] as well as in regulation of macrophage infiltration thereby facilitating PDAC promotion.

To summarize, autophagy plays a critical role in PDAC metabolism and progression through catabolic degradation of bioenergetic macromolecules and consequently supporting oncogenic events and metabolic adaption-driven pathways required for PDAC survival and proliferation in stressful environmental conditions.

\section{MACROPINOCYTOSIS: AN OLD MECHANISM WITH A NEW FUNCTION}

Cell metabolism adaptability is required for pancreatic cancer to survive in an environment where nutrients and oxygen are scarce. Autophagy is a fundamental pathway to produce nutrients and recycle macromolecules, leading to tumor survival in an exceptionally harsh environment. However, it can't be used by the cells to increase their biomass since autophagy is limited by degradation of intracellular content. Interestingly, PDAC cells also rely on a second lysosomal-dependent pathway of macropinocytosis to fuel their elevated metabolic demand and create a net increase in nutrients availability (Figure 1).

One of the main signaling pathways involved in the induction of macropinocytosis is the MAPK pathway. Indeed, PDAC cells carrying a mutant form of KRAS utilize macropinocytosis to incorporate many different nutrients from exogenous origins [41]. For example, tumor cells may use extracellular albumin, since it is the most abundant protein in our blood, or collagen from the extracellular matrix $(\mathrm{ECM})$ to produce glutamine and proline respectively, to support TCA and create the carbon skeleton used for biosynthetic processes $[42,43]$. It has been shown that Ras-driven cancers also rely on this mechanism for lipid scavenging, an advantageous process as lipid synthesis is one of the most expensive metabolic pathways for the cells, in terms of oxygen and energy (ATP and NADPH) [41, 44].

The potent ability of the KRAS mutation to stimulate macropinocytosis may be one of the reasons why it is predominantly mutated in pancreatic cancers [6]. However, although KRAS accelerates the rate of the internalization of extracellular fluid into the endosomal compartment, it does not determine lysosomal degradation of the ingested proteins. Indeed, this second step is strictly controlled by the mTORC1 complex. If the essential amino acids are present in the extracellular microenvironment, tumor cells can use transporters to uptake them for their metabolic de- 
mand. In these conditions, mTORC1 is activated by the high level of nutrients and localizes to the lysosomes, where it prevents the fusion between endosomes and lysosomes, thus limiting the use of extracellular components to fuel the cell metabolism [45]. Instead, in an essential-amino acids depleted microenvironment, mTORC1 is inhibited by the low level of intracellular nutrients and the cells induce lysosomal catabolism of extracellular proteins in order to survive under this limiting condition [46].

The role of mTORC1 is controversial, it is believed to play a central role in tumor growth, but, in a condition of low nutrients, the cells should be able to suppress the mTORC1 signaling pathway in order to survive. This could be a possible reason for so few mutations being found directly on mTORC1 complex in PDAC tumors, since with a constant activation of this pathway tumor cells are unable to adapt to nutrient deprivation. Finally, this mechanism could also be at the base of the modest successes of mTOR inhibitors, especially in KRAS driven cancer. Indeed, rapamycin analogue treatment could enhance the digestion of extracellular content, supporting cell survival and growth in a nutrient-depleted condition $[45,46]$.

\section{EXPLORING NEW THERAPEUTIC PERSPECTIVES}

Most of the patients are diagnosed during advanced stages of pancreatic cancer or the early stages of PDAC are usually asymptomatic due to which the overall survival of patients remained unchanged for decades. Only a modest improvement in overall survival was obtained with FOLFIRINOX (folinic acid, 5-fluorouracil, irinotecan, oxaliplatin) or nab-paclitaxel plus gemcitabine chemotherapy [47]. The impairment in drug delivery due to desmoplasia as well as the fast emergence of resistance consequently limit the efficacy of currently available therapies. Therefore, novel strategies of therapeutic interventions are of utmost importance (Figure 1).

\section{Targeting the metabolic rewiring}

To meet their demand for energy, cancer cells reorganize their entire metabolism (in particular, glycolysis and the TCA cycle) to augment anabolic reactions linked to cell growth and proliferation. This metabolic reprogramming, including increased glycolysis, TCA cycle and autophagy, represents not only a mechanism for survival but also a putative Achille's heel of pancreatic cancer for therapeutic intervention. Emerging therapeutic strategies include the targeting of key regulators of metabolism, such as pyruvate kinase isoenzyme M2 (PKM2) or lactate transport and autophagy. Inhibition of PKM2, a hub for glycolytic switching, reduced cancer cell growth in vitro [48]. The targeting of the lactate production and transport represents another strategy. Although several LDH inhibitors are in preclinical development, their short half-life has limited their efficacy in vivo. On the contrary, AZD3965, a MCT-1 lactate transporter inhibitor, is currently being evaluated in a Phase I trial (ClinicalTrials.gov Identifier NCT01791595) for its ability to reduce tumor growth.
Besides anaerobic glycolysis, the metabolism of NAD, a crucial cofactor in redox balance, is critical for pancreatic cancer development [50]. Inhibition of NAD synthase via NAMPT pathway blocks the cell growth and survival of pancreatic tumor both in vitro and in vivo. The consequent massive reduction in the metabolic activity is accompanied by low levels of NAD, glycolytic flux, lactate production, mitochondrial function and levels of ATP [50]. Abrogating the regeneration of NAD by inhibiting nicotinamide phosphoribosyltransferase disrupts aerobic glycolysis and leads to pancreatic cancer cell growth inhibition in vitro and in vivo, although no tumor regressions were seen in in vivo models.

\section{Autophagy inhibitors}

It has been demonstrated that the genetic and pharmacological inhibition of autophagy delays tumor development in mouse models. One of the most promising drugs appears to be hydroxychloroquine, an autophagy inhibitor approved by the FDA for the treatment of malaria but also commonly used for rheumatic diseases. As a single agent, hydroxychloroquine proved to be inconsistent at autophagy inhibition and demonstrated negligible therapeutic efficacy in patients with pancreatic cancer [49]. However, the combination of hydroxychloroquine with nab-paclitaxel plus gemcitabine chemotherapy is currently undergoing evaluation both in the neoadjuvant setting (ClinicalTrials.gov Identifier NCT01978184) and in advanced-stage disease (ClinicalTrials.gov Identifier NCT01506973; NCT01128296; NCT01494155; NCT01273805).

Given the role of mTOR as a master regulator of autophagy, mTOR inhibitors represented an alternative approach, but, despite the first promising studies, phase II clinical trial failed to demonstrate clinical benefits [50]. Probably, a stronger antitumor effect could result from the inhibition of multiple signaling pathways, as illustrated by the studies on Metformin; an inhibitor of AMPK/mTOR, MAPK, IGF-IR, NFkB pathways. Metformin, a FDA-approved antihyperglycemic drug, is used as first-line therapy for diabetes mellitus type 2 . Thanks to the inhibition of de novo fatty acid synthesis and the alteration of the PPP and subsequent nucleotides and DNA synthesis, Metformin reduces the risk of PDAC in diabetic patients and has antitumor effects [52]. On the contrary, a randomized phase II trial failed to show a survival benefit for simvastatine, an inhibitor of cholesterol synthesis, in patients treated with gemcitabine [51].

On the other hand, the reduction in cholesterol uptake through Low-Density Lipoprotein Receptor (LDLR) blockade may be an alternative strategy, as it sensitizes cells to chemotherapeutic drugs such as gemcitabine [53].

\section{Macropinocytosis inhibitors}

As previously described, micropinocytosis is adopted by pancreatic cancer in order to sustain tumor propagation. Indeed, this survival mechanism may represent an avenue for novel therapeutics in PDAC.

The currently available pharmacological tools to inhibit macropinocytosis include PI3K-AKT-mTOR inhibitors, actin 
polymerization inhibitors [54], and sodium/hydrogen exchangers (NHEs) inhibitors [55].

The PI3K inhibitors wortmannin and LY290042 are known to block macropinocytosis and phagocytosis in different cell types [56]. Given the involvement of mTOR in lysosomal degradation and promotion of macropinocytosis, rapamycin analogs could represent a treatment option. Another therapeutic approach includes the disruption of cancer cell metabolic activity through inhibition of the phosphoinositide-3-kinase (PI3K) pathway. It was demonstrated that the class I PI3K inhibitor wortmannin inhibits scission of macropinosomes from the cell surface, reducing pancreatic cancer motility, invasion and metastasis [57].

The actin perturbant cytochalasin $D$ and latrunculins are known to inhibit not only membrane but also several other cellular processes involving actin polymerization, resulting in an aspecific targeting of macropinocytosis [58]. Currently, the selective NHE blockerethyl-isopropyl amiloride (EIPA) and di-methyl amiloride are considered to be the first choices for pharmacological inhibition of macropinocytosis [56]. However, possible endocytosisunrelated effects of these drugs on ion transport, intracellular $\mathrm{pH}$ and cytoskeleton limit their use as pharmacological inhibitors of macropinocytosis.

In addition, a recent study suggests that the concomitant targeting of the glutamine metabolism and macropinocytosis may provide an appropriate therapeutic rationale for KRAS-driven tumors [59]. On the other hand, macropinocytosis can be positively used to enhance the internalization of nanoparticles, carrying small molecules, genetic components or chemotherapeutic drugs [42].

\section{CONCLUDING REMARKS}

The toxicity profile of drugs targeting ubiquitous metabolic enzymes in humans is a major concern. To enable targeting of metabolic treatments and to ensure an adequate toxicity profile, identification of mutations that result in metabolic pathway addiction in cancer cells will be crucial. For example, gemcitabine resistance emergences for the upregulation of genes involved in pyrimidine biosynthesis and

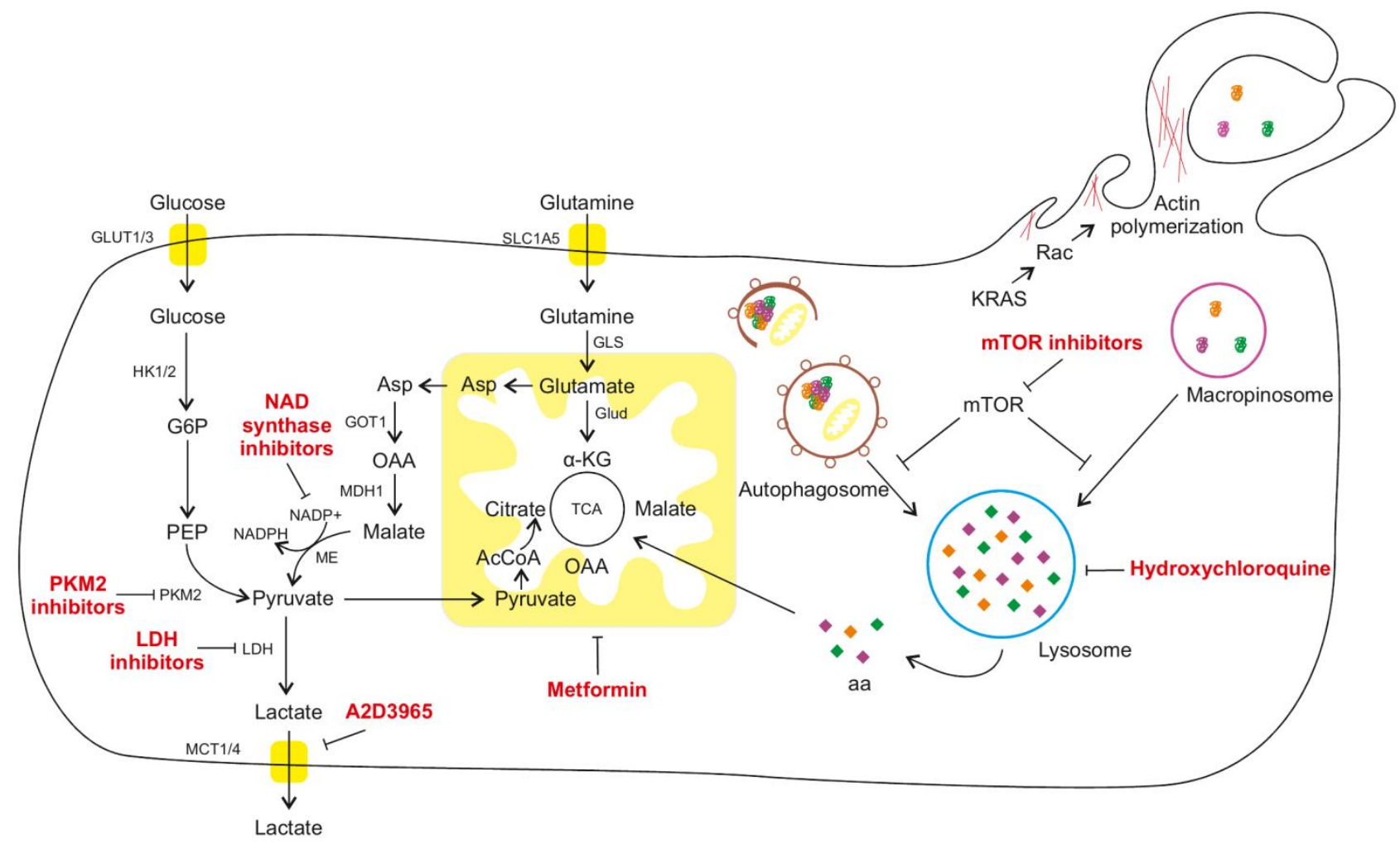

FIGURE 1: Macroautophagy and macropinocytosis as pro-survival mechanisms to sustain cancer cell metabolism in a nutrient limiting environment. Under nutrient rich conditions, cancer cells take up glucose and amino acids through membrane transporters and they can be used to support glycolysis and tricarboxylic acid (TCA), producing ATP and intermediates for anabolic processes. However, PDAC is completely surrounded by a fibrotic barrier, thus limiting the nutrients' delivery to the tumor cells. For this reason, PDAC cells develop two different lysosomal degrading mechanisms. Macroautophagy is a catabolic degradation of damaged cytoplasmic components (organelles and macromolecules), while macropinocytosis is a catabolic degradation of extracellular components previously internalized by the cell. Both mechanisms can produce amino acids and other nutrients that can fuel cell metabolism and sustain survival and proliferation. $\alpha$-KG: $\alpha$-ketoglutarate; AcCoA: acetyl coenzyme A; Asp: Aspartate; G6P: glucose-6-phosphate; GOT1: Glutamic-oxaloacetic transaminase 1; GLS: Glutaminase; GLUD: Glutamate Dehydrogenase; GLUT: Glucose Transporter; HK 1/2: hexokinase 1/2; LDH: lactate dehydrogenase; MCT 1/4: Monocarboxylate transporter 1/4; MDH1: Malate Dehydrogenase 1; ME: Malate dehydrogenase; mTOR: mammalian target of rapamycin; NADPH: nicotinamide adenine dinucleotide phosphate; OAA: oxaloacetate; PEP: Phosphoenolpyruvate; PKM2: Pyruvate kinase isozymes M2; SLC1A5: Solute Carrier Family 1, member 5; TCA: tricarboxylic acid. 
HIF overexpression. Inhibition of pyrimidine synthesis and HIF through leflunomide and digoxin respectively, would disable DNA synthesis as well as adaptation to hypoxia, thereby rendering pancreatic cancer sensitive to presumably any therapeutic stress (sensitizing PDAC tumors to gemcitabine) [60]. The inhibition of glutamine metabolism, which regulates redox balance, can sensitize PDAC to oxidative stress, generated by standard chemotherapy used in PDAC patients. These findings can pave the way to new therapeutic strategies for the synergistic application of glutamine inhibitors and therapies able to increase reactive oxygen species (ROS) production, as radiotherapy and chemotherapy [18].

Therapeutic resistance of PDAC to radiotherapy, targeted agents, and chemotherapy means that new therapeutic strategies are urgently needed. Recently, several studies in cancer therapy are exploiting the biochemical differences between cancer cell and normal cell metabolism. The metabolism of pancreatic cancer is profoundly rewired by KRAS mutations, offering new opportunities for selective targeting. Therefore, one avenue would be to target pancreatic cancer nutrient acquisition processes, as a number of studies have linked autophagy and micropinocytosis to tumor survival and progression. While autophagy is a potent and adaptive survival mechanism, cells are ultimately limited by intracellular content. In contrast, macropinocytosis is a way to generate a diverse array of essential building blocks from an exogenous source which can contribute to cellular biomass. Therefore both these processes are crucial for pancreatic cancer cells, providing them with a means to grow in the exceptionally harsh PDAC environment. In addition, considering that these

\section{REFERENCES}

1. Siegel RL, Miller KD, Jemal A (2018). Cancer statistics, 2018. CA Cancer J Clin 68(1): 7-30. doi: 10.3322/caac.21442

2. Waddell N, Pajic M, Patch AM, Chang DK, Kassahn KS, Bailey P, Johns AL, Miller D, Nones K, Quek K, Quinn MC, Robertson AJ, Fadlullah MZ, Bruxner TJ, Christ AN, Harliwong I, Idrisoglu S, Manning S, Nourse C, Nourbakhsh E, Wani S, Wilson PJ, Markham E, Cloonan N, Anderson MJ, Fink JL, Holmes O, Kazakoff SH, Leonard C, Newell F, et al. (2015). Whole genomes redefine the mutational landscape of pancreatic cancer. Nature 518(7540): 495-501. doi: 10.1038/nature14169

3. Guerra C, Barbacid M (2013). Genetically engineered mouse models of pancreatic adenocarcinoma. Mol Oncol 7(2): 232-247. doi: 10.1016/j.molonc.2013.02.002

4. Vander Heiden MG, Cantley LC, Thompson CB (2009). Understanding the Warburg effect: the metabolic requirements of cell proliferation. Science 324(5930): 1029-1033. doi: 10.1126/science.1160809

5. Bryant KL, Mancias JD, Kimmelman AC, Der CJ (2014). KRAS: feeding pancreatic cancer proliferation. Trends Biochem Sci 39(2): 91-100. doi: 10.1016/j.tibs.2013.12.004

6. Biancur DE, Kimmelman AC (2018). The plasticity of pancreatic cancer metabolism in tumor progression and therapeutic resistance. Biochim Biophys Acta. doi: 10.1016/j.bbcan.2018.04.011

7. Buckley CM, King JS (2017). Drinking problems: mechanisms of macropinosome formation and maturation. FEBS J 284(22): 3778 3790. doi: 10.1111/febs.14115 pathways converge at the lysosome, it is also tempting to speculate that lysosomal perturbation could be particularly toxic in PDAC tumors.

Future studies will be needed to gather a more detailed understanding of the molecular events controlling nutrient acquisition in pancreatic cancer. Moreover, studies focused on combination therapies employing autophagy/macropinocytosis inhibitors in conjunction with other metabolic pathway inhibitors could pave the way to improve therapeutic outcomes.

\section{ACKNOWLEDGMENTS}

We would like to thank Dr. Paolo Ettore Porporato and Albert Mackintosh for critically reading of the manuscript and for helpful discussions. AD is supported by the Marie Curie Innovative Training Network (ITN).

\section{CONFLICT OF INTEREST}

The authors declare no conflict of interest.

\section{COPYRIGHT}

(C) 2018 Derle et al. This is an open-access article released under the terms of the Creative Commons Attribution (CC $\mathrm{BY}$ ) license, which allows the unrestricted use, distribution, and reproduction in any medium, provided the original author and source are acknowledged.

Please cite this article as: Abhishek Derle, Maria Chiara De Santis, Luca Gozzelino, Edoardo Ratto and Miriam Martini (2018). The role of metabolic adaptation to nutrient stress in pancreatic cancer. Cell Stress 2(12): 332-339. doi: 10.15698/cst2018.12.166

8. Ryan DP, Hong TS, Bardeesy N (2014). Pancreatic adenocarcinoma. N Engl J Med 371(22): 2140-2141. doi: 10.1056/NEJMc1412266

9. Ying $\mathrm{H}$, Kimmelman AC, Lyssiotis CA, Hua S, Chu GC, FletcherSananikone E, Locasale JW, Son J, Zhang H, Coloff JL, Yan H, Wang W, Chen S, Viale A, Zheng $H$, Paik JH, Lim C, Guimaraes AR, Martin ES, Chang J, Hezel AF, Perry SR, Hu J, Gan B, Xiao Y, Asara JM, Weissleder $R$, Wang YA, Chin L, Cantley LC, et al. (2012). Oncogenic Kras maintains pancreatic tumors through regulation of anabolic glucose metabolism. Cell 149(3): 656-670. doi: 10.1016/j.cell.2012.01.058

10. Zhou W, Capello M, Fredolini C, Piemonti L, Liotta LA, Novelli F, Petricoin EF (2011). Proteomic analysis of pancreatic ductal adenocarcinoma cells reveals metabolic alterations. J Proteome Res 10(4): 1944-1952. doi: 10.1021/pr101179t

11. Jiang $P$, Du W, Wang X, Mancuso A, Gao X, Wu M, Yang X (2011). p53 regulates biosynthesis through direct inactivation of glucose-6phosphate dehydrogenase. Nat Cell Biol 13(3): 310-316. doi: $10.1038 /$ ncb2172

12. De Santis MC, Porporato PE, Martini M, Morandi A (2018). Signaling Pathways Regulating Redox Balance in Cancer Metabolism. Frontiers in oncology 8(126. doi: 10.3389/fonc.2018.00126.

13. Guillaumond F, Leca J, Olivares $O$, Lavaut MN, Vidal N, Berthezene P, Dusetti NJ, Loncle C, Calvo E, Turrini O, lovanna JL, Tomasini R, Vasseur S (2013). Strengthened glycolysis under hypoxia supports tumor symbiosis and hexosamine biosynthesis in pancreatic adeno- 
carcinoma. Proc Natl Acad Sci U S A 110(10): 3919-3924. doi: 10.1073/pnas.1219555110

14. Kim JW, Tchernyshyov I, Semenza GL, Dang CV (2006). HIF-1mediated expression of pyruvate dehydrogenase kinase: a metabolic switch required for cellular adaptation to hypoxia. Cell Metab 3(3): 177-185. doi: 10.1016/j.cmet.2006.02.002

15. Patra KC, Hay N (2014). The pentose phosphate pathway and cancer. Trends Biochem Sci 39(8): 347-354. doi: 10.1016/j.tibs.2014.06.005

16. Blum R, Kloog $Y$ (2014). Metabolism addiction in pancreatic cancer. Cell Death Dis 5:e1065. doi: 10.1038/cddis.2014.38

17. Wise DR, Thompson CB (2010). Glutamine addiction: a new therapeutic target in cancer. Trends Biochem Sci 35(8): 427-433. doi: 10.1016/j.tibs.2010.05.003

18. Son J, Lyssiotis CA, Ying H, Wang X, Hua S, Ligorio M, Perera RM, Ferrone $C R$, Mullarky E, Shyh-Chang $N$, Kang $Y$, Fleming JB, Bardeesy $\mathrm{N}$, Asara JM, Haigis MC, DePinho RA, Cantley LC, Kimmelman AC (2013). Glutamine supports pancreatic cancer growth through a KRASregulated metabolic pathway. Nature 496(7443): 101-105. doi: 10.1038/nature12040

19. Yang A, Herter-Sprie G, Zhang H, Lin EY, Biancur D, Wang X, Deng J, Hai J, Yang S, Wong KK, Kimmelman AC (2018). Autophagy Sustains Pancreatic Cancer Growth through Both Cell-Autonomous and Nonautonomous Mechanisms. Cancer Discov 8(3): 276-287. doi: 10.1158/2159-8290.cd-17-0952

20. Greer EL, Brunet A (2005). FOXO transcription factors at the interface between longevity and tumor suppression. Oncogene 24(50): 7410-7425. doi: 10.1038/sj.onc.1209086

21. Yang S, Hwang S, Kim M, Seo SB, Lee JH, Jeong SM (2018). Mitochondrial glutamine metabolism via GOT2 supports pancreatic cancer growth through senescence inhibition. Cell Death Dis 9(2): 55 . doi: 10.1038/s41419-017-0089-1

22. Semenza GL (2010). HIF-1: upstream and downstream of cancer metabolism. Curr Opin Genet Dev 20(1): 51-56. doi: 10.1016/j.gde.2009.10.009

23. Payen VL, Porporato PE, Baselet B, Sonveaux P (2016). Metabolic changes associated with tumor metastasis, part 1: tumor $\mathrm{pH}$, glycolysis and the pentose phosphate pathway. Cell Mol Life Sci 73(7): 13331348. doi: 10.1007/s00018-015-2098-5

24. Sousa CM, Biancur DE, Wang X, Halbrook CJ, Sherman MH, Zhang L, Kremer D, Hwang RF, Witkiewicz AK, Ying H, Asara JM, Evans RM, Cantley LC, Lyssiotis CA, Kimmelman AC (2016). Pancreatic stellate cells support tumour metabolism through autophagic alanine secretion. Nature 536: 479. doi: 10.1038/nature19084

25. Cianfanelli V, Fuoco C, Lorente M, Salazar M, Quondamatteo F, Gherardini PF, De Zio D, Nazio F, Antonioli M, D'Orazio M, Skobo T, Bordi M, Rohde M, Dalla Valle L, Helmer-Citterich M, Gretzmeier C, Dengjel J, Fimia GM, Piacentini M, Di Bartolomeo S, Velasco G, Cecconi $F$ (2015). AMBRA1 links autophagy to cell proliferation and tumorigenesis by promoting c-Myc dephosphorylation and degradation. Nat Cell Biol 17(1): 20-30. doi: 10.1038/ncb3072

26. Cicchini M, Chakrabarti R, Kongara S, Price S, Nahar R, Lozy F, Zhong $\mathrm{H}$, Vazquez A, Kang $\mathrm{Y}$, Karantza $\mathrm{V}$ (2014). Autophagy regulator BECN1 suppresses mammary tumorigenesis driven by WNT1 activation and following parity. Autophagy 10(11): 2036-2052. doi: 10.4161/auto.34398

27. Lazova R, Camp RL, Klump V, Siddiqui S, Amaravadi R, Pawelek JM (2011). Punctate LC3B expression is a common feature of solid tumors and associated with proliferation, metastasis and poor outcome. Clin Cancer Res 18(2):370-9. doi: 10.1158/1078-0432.ccr-11-1282
28. Mikhaylova O, Stratton $Y$, Hall D, Kellner E, Ehmer B, Drew Angela F, Gallo Catherine A, Plas David R, Biesiada J, Meller J, Czyzyk-Krzeska Maria F (2012). VHL-Regulated MiR-204 Suppresses Tumor Growth through Inhibition of LC3B-Mediated Autophagy in Renal Clear Cell Carcinoma. Cancer Cell 21(4): 532-546. doi: 10.1016/j.ccr.2012.02.019

29. Yang S, Wang X, Contino G, Liesa M, Sahin E, Ying H, Bause A, Li Y, Stommel JM, Dell'antonio G, Mautner J, Tonon G, Haigis M, Shirihai OS, Doglioni C, Bardeesy N, Kimmelman AC (2011). Pancreatic cancers require autophagy for tumor growth. Genes Dev 25(7): 717-729. doi: 10.1101/gad.2016111

30. Rosenfeldt MT, O'Prey J, Morton JP, Nixon C, Mackay G, Mrowinska A, Au A, Rai TS, Zheng L, Ridgway R, Adams PD, Anderson $\mathrm{KI}$, Gottlieb E, Sansom OJ, Ryan KM (2013). p53 status determines the role of autophagy in pancreatic tumour development. Nature 504: 296. doi: $10.1038 /$ nature 12865

31. Ko YH, Cho YS, Won HS, Jeon EK, An HJ, Hong SU, Park JH, Lee MA (2013). Prognostic significance of autophagy-related protein expression in resected pancreatic ductal adenocarcinoma. Pancreas 42(5): 829-835. doi: 10.1097/MPA.0b013e318279d0dc

32. Liang C, Shi S, Meng Q, Liang D, Ji S, Zhang B, Qin Y, Xu J, Ni Q, Yu X (2017). Complex roles of the stroma in the intrinsic resistance to gemcitabine in pancreatic cancer: where we are and where we are going. Exp Mol Med 49:e406. doi: 10.1038/emm.2017.255

33. Papademetrio DL, Lompardía SL, Simunovich T, Costantino S, Mihalez CY, Cavaliere V, Álvarez É (2016). Inhibition of Survival Pathways MAPK and NF-kB Triggers Apoptosis in Pancreatic Ductal Adenocarcinoma Cells via Suppression of Autophagy. Targeted Oncology 11(2): 183-195. doi: 10.1007/s11523-015-0388-3

34. Li L, Chen H, Gao Y, Wang Y-W, Zhang G-Q, Pan S-H, Ji L, Kong R, Wang G, Jia Y-H, Bai X-W, Sun B (2016). Long Noncoding RNA MALAT1 Promotes Aggressive Pancreatic Cancer Proliferation and Metastasis via the Stimulation of Autophagy. Mol Cancer Ther 5(9): 2232-43. doi: 10.1158/1535-7163.mct-16-0008

35. Zhu H, Wang D, Zhang L, Xie X, Wu Y, Liu Y, Shao G, Su Z (2014). Upregulation of autophagy by hypoxia-inducible factor-1alpha promotes EMT and metastatic ability of CD133+ pancreatic cancer stemlike cells during intermittent hypoxia. Oncol Rep 32(3): 935-942. doi: 10.3892/or.2014.3298

36. Perera RM, Stoykova S, Nicolay BN, Ross KN, Fitamant J, Boukhali $M$, Lengrand J, Deshpande V, Selig MK, Ferrone CR, Settleman J, Stephanopoulos G, Dyson NJ, Zoncu R, Ramaswamy S, Haas W, Bardeesy N (2015). Transcriptional control of autophagy-lysosome function drives pancreatic cancer metabolism. Nature 524(7565): 361-365. doi: $10.1038 /$ nature14587

37. Klein K, Werner K, Teske C, Schenk M, Giese T, Weitz J, Welsch T (2016). Role of TFEB-driven autophagy regulation in pancreatic cancer treatment. Int J Oncol 49(1): 164-172. doi: 10.3892/ijo.2016.3505

38. Maertin S, Elperin JM, Lotshaw E, Sendler M, Speakman SD, Takakura K, Reicher BM, Mareninova OA, Grippo PJ, Mayerle J, Lerch MM, Gukovskaya AS (2017). Roles of autophagy and metabolism in pancreatic cancer cell adaptation to environmental challenges. Am J Physiol Gastrointest Liver Physiol 313(5): G524-g536. doi: 10.1152/ajpgi.00138.2017

39. Wyart E, Reano S, Hsu MY, Longo DL, Li M, Hirsch E, Filigheddu N, Ghigo A, Riganti C, Porporato PE (2018). Metabolic Alterations in a Slow-Paced Model of Pancreatic Cancer-Induced Wasting. Oxid Med Cell Longev 2018: 6419805. doi: 10.1155/2018/6419805

40. Kim SE, Park HJ, Jeong HK, Kim MJ, Kim M, Bae ON, Baek SH (2015). Autophagy sustains the survival of human pancreatic cancer PANC-1 cells under extreme nutrient deprivation conditions. Biochem Biophys Res Commun 463(3): 205-210. doi 10.1016/j.bbrc.2015.05.022 
41. Palm W, Thompson CB (2017). Nutrient acquisition strategies of mammalian cells. Nature 546(7657): 234-242. doi: $10.1038 /$ nature22379

42. Davidson SM, Jonas O, Keibler MA, Hou HW, Luengo A, Mayers JR, Wyckoff J, Del Rosario AM, Whitman M, Chin CR, Condon KJ, Lammers A, Kellersberger KA, Stall BK, Stephanopoulos G, Bar-Sagi D, Han J, Rabinowitz JD, Cima MJ, Langer R, Vander Heiden MG (2017). Direct evidence for cancer-cell-autonomous extracellular protein catabolism in pancreatic tumors. Nat Med 23(2): 235-241. doi: 10.1038/nm.4256

43. Olivares O, Mayers JR, Gouirand V, Torrence ME, Gicquel T, Borge L, Lac S, Roques J, Lavaut MN, Berthezene P, Rubis M, Secq V, Garcia S, Moutardier V, Lombardo D, lovanna JL, Tomasini R, Guillaumond F, Vander Heiden MG, Vasseur S (2017). Collagen-derived proline promotes pancreatic ductal adenocarcinoma cell survival under nutrient limited conditions. Nat Commun 8: 16031. doi 10.1038/ncomms16031

44. Danai LV, Babic A, Rosenthal MH, Dennstedt EA, Muir A, Lien EC, Mayers JR, Tai K, Lau AN, Jones-Sali P, Prado CM, Petersen GM, Takahashi N, Sugimoto M, Yeh JJ, Lopez N, Bardeesy N, Fernandez-Del Castillo C, Liss AS, Koong AC, Bui J, Yuan C, Welch MW, Brais LK, Kulke $\mathrm{MH}$, Dennis C, Clish CB, Wolpin BM, Vander Heiden MG (2018). Altered exocrine function can drive adipose wasting in early pancreatic cancer. Nature 558(7711): 600-604. doi: 10.1038/s41586-018-0235-7

45. Thompson CB, Palm W (2016). Reexamining How Cancer Cells Exploit the Body's Metabolic Resources. Cold Spring Harb Symp Quant Biol 81: 67-72. doi: 10.1101/sqb.2016.81.030734

46. Palm W, Park Y, Wright K, Pavlova NN, Tuveson DA, Thompson CB (2015). The Utilization of Extracellular Proteins as Nutrients Is Suppressed by mTORC1. Cell 162(2): 259-270. doi: 10.1016/j.cell.2015.06.017

47. Garrido-Laguna I, Hidalgo M (2015). Pancreatic cancer: from stateof-the-art treatments to promising novel therapies. Nat Rev Clin Oncol 12(6): 319-334. doi: 10.1038/nrclinonc.2015.53

48. Li X, Deng S, Liu M, Jin Y, Zhu S, Chen J, He C, Qin Q, Wang C, Zhao $\mathrm{G}$ (2018). The responsively decreased PKM2 facilitates the survival of pancreatic cancer cells in hypoglucose. Cell Death Dis 9(2): 133. doi: 10.1038/s41419-017-0158-5

49. Wolpin BM, Rubinson DA, Wang $X$, Chan JA, Cleary JM, Enzinger PC, Fuchs CS, McCleary NJ, Meyerhardt JA, Ng K, Schrag D, Sikora AL, Spicer BA, Killion L, Mamon H, Kimmelman AC (2014). Phase II and pharmacodynamic study of autophagy inhibition using hydroxychloroquine in patients with metastatic pancreatic adenocarcinoma. Oncologist 19(6): 637-638. doi: 10.1634/theoncologist.2014-0086

50. Javle MM, Shroff RT, Xiong H, Varadhachary GA, Fogelman D, Reddy SA, Davis D, Zhang Y, Wolff RA, Abbruzzese JL (2010). Inhibition of the mammalian target of rapamycin (mTOR) in advanced pancreatic cancer: results of two phase II studies. BMC Cancer 10: 368. doi: 10.1186/1471-2407-10-368

51. Hong JY, Nam EM, Lee J, Park JO, Lee SC, Song SY, Choi SH, Heo JS, Park SH, Lim HY, Kang WK, Park YS (2014). Randomized double- blinded, placebo-controlled phase II trial of simvastatin and gemcitabine in advanced pancreatic cancer patients. Cancer Chemother Pharmacol 73(1): 125-130. doi: 10.1007/s00280-013-2328-1

52. Cantoria MJ, Boros LG, Meuillet EJ (2014). Contextual inhibition of fatty acid synthesis by metformin involves glucose-derived acetyl-CoA and cholesterol in pancreatic tumor cells. Metabolomics 10(1): 91104. doi: 10.1007/s11306-013-0555-4

53. Guillaumond F, Bidaut G, Ouaissi M, Servais S, Gouirand V, Olivares O, Lac S, Borge L, Roques J, Gayet O, Pinault M, Guimaraes C, Nigri J, Loncle C, Lavaut MN, Garcia S, Tailleux A, Staels B, Calvo E, Tomasini R, lovanna JL, Vasseur S (2015). Cholesterol uptake disruption, in association with chemotherapy, is a promising combined metabolic therapy for pancreatic adenocarcinoma. Proc Natl Acad Sci U S A 112(8): 2473-2478. doi: 10.1073/pnas.1421601112

54. Aleksandrowicz P, Marzi A, Biedenkopf N, Beimforde N, Becker S, Hoenen T, Feldmann $H$, Schnittler HJ (2011). Ebola virus enters host cells by macropinocytosis and clathrin-mediated endocytosis. J Infect Dis 204 (Suppl 3): S957-967. doi: 10.1093/infdis/jir326

55. Koivusalo M, Welch C, Hayashi H, Scott CC, Kim M, Alexander T, Touret N, Hahn KM, Grinstein S (2010). Amiloride inhibits macropinocytosis by lowering submembranous $\mathrm{pH}$ and preventing Rac1 and Cdc42 signaling. J Cell Biol 188(4): 547-563. doi: 10.1083/jcb. 200908086

56. Ivanov Al (2008). Pharmacological inhibition of endocytic pathways: is it specific enough to be useful? Methods Mol Biol 440: 15-33. doi: 10.1007/978-1-59745-178-9_2

57. Teranishi F, Takahashi N, Gao N, Akamo Y, Takeyama H, Manabe T, Okamoto T (2009). Phosphoinositide 3-kinase inhibitor (wortmannin) inhibits pancreatic cancer cell motility and migration induced by hyaluronan in vitro and peritoneal metastasis in vivo. Cancer Sci 100(4): 770-777. doi: 10.1111/j.1349-7006.2009.01084.x

58. Stockinger W, Zhang SC, Trivedi V, Jarzylo LA, Shieh EC, Lane WS, Castoreno $A B$, Nohturfft $A$ (2006). Differential requirements for actin polymerization, calmodulin, and $\mathrm{Ca} 2+$ define distinct stages of lysosome/phagosome targeting. Mol Biol Cell 17(4): 1697-1710. doi: 10.1091/mbc.e05-12-1140

59. Seo JW, Choi J, Lee SY, Sung S, Yoo HJ, Kang MJ, Cheong H, Son J (2016). Autophagy is required for PDAC glutamine metabolism. Sci Rep 6: 37594. doi: 10.1038/srep37594

60. Shukla SK, Purohit V, Mehla K, Gunda V, Chaika NV, Vernucci E, King RJ, Abrego J, Goode GD, Dasgupta A, Illies AL, Gebregiworgis T, Dai B, Augustine JJ, Murthy D, Attri KS, Mashadova O, Grandgenett PM, Powers R, Ly QP, Lazenby AJ, Grem JL, Yu F, Mates JM, Asara JM Kim JW, Hankins JH, Weekes C, Hollingsworth MA, Serkova NJ, et al. (2017). MUC1 and HIF-1alpha Signaling Crosstalk Induces Anabolic Glucose Metabolism to Impart Gemcitabine Resistance to Pancreatic Cancer. Cancer Cell 32(1): 71-87 e77. doi: 10.1016/j.ccell.2017.06.004 